\title{
Accuracy of Magnetic Resonance Parkinsonism Index in Differentiating Progressive Supranuclear Palsy from Parkinson's Disease among South Indian Population: A Retrospective Case Control Study
}

\author{
Vasanthapriya Janarthanan ${ }^{1}$ Kulasekaran Nadhamuni ${ }^{2}$ Sibhithran Rajakumar ${ }^{2}$ \\ Elamparidhi Padmanaban ${ }^{2}$ Umamageswari Amirthalingam² ${ }^{2}$ Yashkumar Achantani ${ }^{3}$
}

\footnotetext{
${ }^{1}$ Department of Radiology and Medical Imaging, Sri Venkateshwaraa Medical College Hospital \& Research Centre, Puducherry, India

2 Department of Radio-Diagnosis, Sri Manakula Vinayagar Medical College and Hospital, Puducherry, India

3 Rajsheel Diagnostics, Dabra, Gwalior, India

Indian J Radiol Imaging 2021;31:596-600.
}

\begin{abstract}
Address for correspondence Sibhithran Rajakumar, MBBS, MD (RD), 9, Annai Teresa Street, Annai Indira Nagar, Velachery, Chennai, Tamil Nadu 600 042, India (e-mail: sibhius@gmail.com).
\end{abstract}

\begin{abstract}
Keywords

- magnetic resonance Parkinsonism index

- midbrain/pons ratio

- progressive supranuclear palsy

- Parkinson's disease
\end{abstract}

Context Progressive supranuclear palsy (PSP) is a neurodegenerative disorder which comes under Parkinsonism plus syndrome. As this spectrum of disease has many overlapping clinical as well as imaging findings, some quantitative parameters like magnetic resonance Parkinsonism index and midbrain/pons ratio are useful to differentiate PSP from other PD patients.

Aims The study aimed to detect sensitivity and specificity of magnetic resonance Parkinsonism index in differentiating PSP from PD.

Settings and Design It was a retrospective case-control study conducted in Sri Manankula Vinayagar Medical College, Puducherry, during the period of January 2018 to June 2019.

Materials and Methods The 87 subjects, who were diagnosed and grouped into three categories (PSP, PD, and control) after performing magnetic resonance imaging brain, were reviewed. The parameters like the area of Pons and midbrain, width of MCP and $\mathrm{SCP}, \mathrm{P} / \mathrm{M}, \mathrm{M} / \mathrm{P}$, and MRPI were calculated.

Statistical Analysis One-way ANOVA and Chi-square test was used. The sensitivity, specificity, diagnostic accuracy, and cut-off values obtained with receiver operating characteristic curve analysis were determined.

Results The mean age of presentation was approximately 75 years with male predominance. The cut-off value of MRPI obtained in this study was 13.4 with $100 \%$ sensitivity and specificity. Even though M/P ratio was found to be statistically significant among PSP patients; cut-off value was not obtained.

Conclusion MRPI was concluded as the better tool in diagnosing PSP compared with the $\mathrm{M} / \mathrm{P}$ ratio. Hence the combined qualitative as well as quantitative measurement of MRPI will increase the diagnostic accuracy of PSP.
DOI https://doi.org/ $10.1055 / \mathrm{s}-0041-1736402$ ISSN 0971-3026.

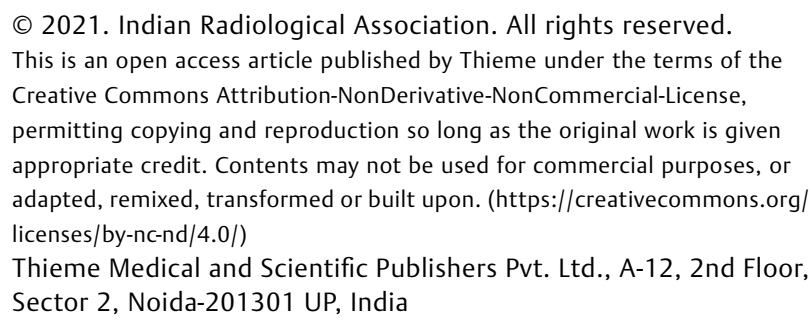




\section{Introduction}

Progressive supranuclear palsy (PSP) is a neurodegenerative disorder, which is diagnosed clinically by postural instability with falls, supranuclear vertical gaze abnormalities with Parkinsonian features, and frontal cognitive disturbances. ${ }^{1}$ Although signs such as vertical gaze palsy is specific for PSP, it is difficult to distinguish PSP from other Parkinson's disease (PD) clinically, which makes it essential to make an early distinction between PSP and other PD. ${ }^{2}$ This research was mainly conducted to study the diagnostic accuracy of magnetic resonance Parkinsonism index (MRPI) and midbrain and Pons ratio ( $\mathrm{M} / \mathrm{P})$ in differentiating PSP patients from PD patients and controls.

\section{Materials and Methods}

The main aim of this study was to detect sensitivity and specificity of MRPI in differentiating PSP from PD. The study was conducted at Sri Manakula Vinayagar Medical College and hospital, Puducherry, which is a multidisciplinary, 900-bedded hospital with fully equipped radiology department during the period of January 2018 to June 2019. It was a retrospective case-control study of magnetic resonance imaging (MRI) brain done in 87 subjects (58 patients and 29 controls), grouped into three categories-26 PSP patients, 32 PD patients, and 29 controls who were found to be neurologically stable (age $>60$ years with no gender predilection).

The MRI images like sagittal T1W sequences (TE approximately 15 , TR approximately $1,400,512 \times 512$ matrix, and $2 \mathrm{~mm}$ slice thickness approximately $2 \mathrm{~mm}$ ) and coronal T2W sequences obtained (TE approximately 125 , TR approximately $8000,512 \times 512$ matrix, and $2 \mathrm{~mm}$ slice thickness) were assessed and parameters like area of pons $(\mathrm{P})$ and midbrain $(\mathrm{M})$, width of middle cerebellar peduncle, and superior cerebellar peduncle (SCP, MCP) were measured.

Mid sagittal T1W images were evaluated for calculating the area of Pons and midbrain ( - Fig. $\mathbf{1 a}$ and $\mathbf{b}$ ). The width of MCP was also calculated from the sagittal T1W echo spin sequence (-Fig. 1c). The width of the SCP was measured from the coronal T2W image ( - Fig. 1d). From these parameters, $\mathrm{M} / \mathrm{P}$ ratio and MRPI were calculated with the formula $(\mathrm{P} / \mathrm{M}) \times(\mathrm{MCP} / \mathrm{SCP})$ for each patient in three categories. ${ }^{3}$

One-way ANOVA was used to assess the association of continuous covariates with the group and Chi-square test was used to test the association of categorical factors with the group. The sensitivity, specificity, and diagnostic accuracy were determined for differentiating PSP from PD and controls by using the optimal cut-off values determined with receiver operating characteristic (ROC) ( - Fig. 2a-c) curve analysis.

\section{Results}

There is no significant statistical difference found in the age distribution among the three groups.

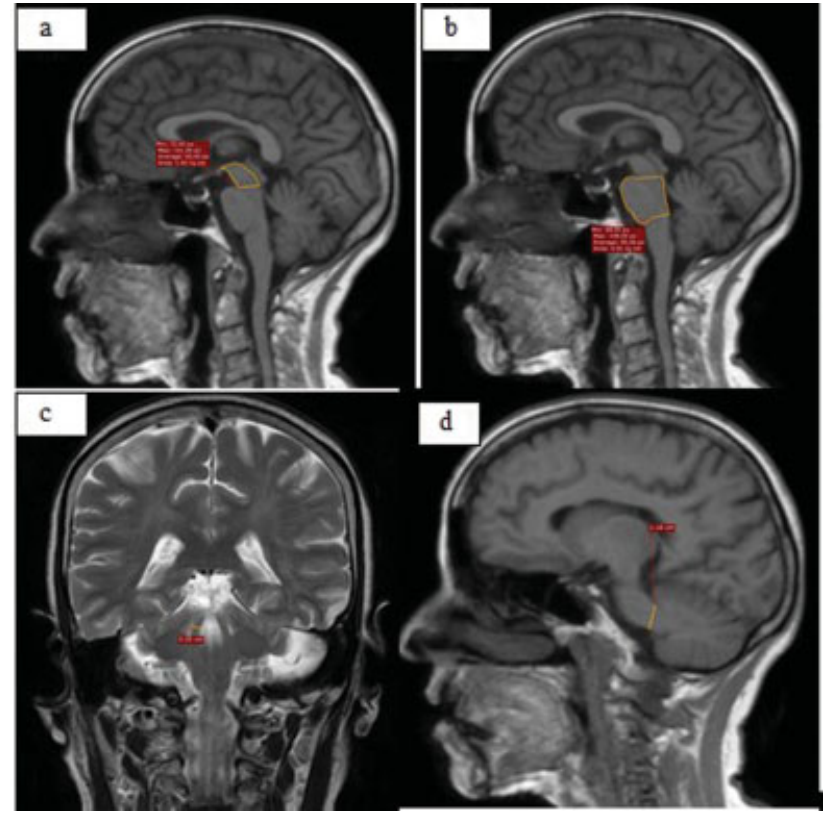

Fig. 1 MRI T1W sagittal image in a control patient showing the midbrain area $\left(1.42 \mathrm{~cm}^{2}\right)(\mathrm{a})$, the area of pons $\left(4.91 \mathrm{~cm}^{2}\right)(\mathrm{b}), \mathrm{T} 2 \mathrm{~W}$ coronal image showing the width of superior cerebellar peduncle $(0.33 \mathrm{~cm})(\mathrm{c})$, and sagittal image shows the middle cerebellar peduncle width $(1.16 \mathrm{~cm})(\mathrm{d})$.
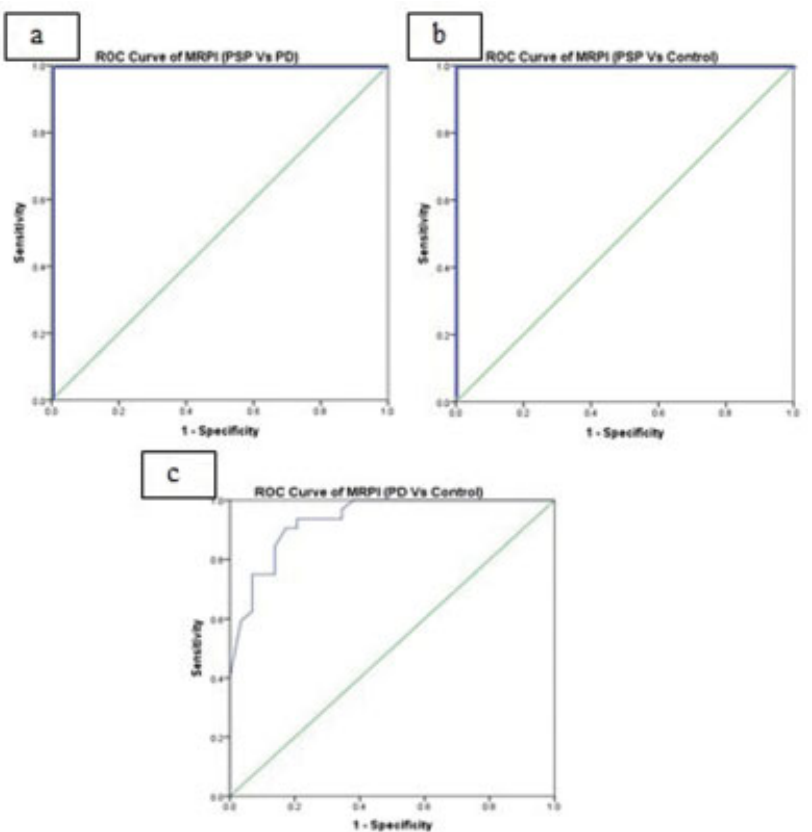

Fig. 2 (a-c ROC curve plotted for MRPI to calculate the cut-off value of MRPI index with respect to various study groups (PSP, PD, control groups). MRPI, magnetic resonance Parkinsonism index; ROC, receiver operating characteristics.

The mean age of distribution of PSP was approximately 75 and 72 years for PD in our study and males (approximately $70 \%$ ) are found to be more commonly affected than females (approximately $30 \%$ ) in all three groups (-Table $\mathbf{1}$ ). 
Table 1 Mean with standard deviation of demographic data of our study population

\begin{tabular}{|l|l|l|l|}
\hline Variables & $\begin{array}{l}\text { PSP } \\
(\boldsymbol{n}=26)\end{array}$ & $\begin{array}{l}\text { PD } \\
(\boldsymbol{n}=32)\end{array}$ & $\begin{array}{l}\text { Control } \\
(\boldsymbol{n}=29)\end{array}$ \\
\hline $\begin{array}{l}\text { Age } \\
\text { distribution }\end{array}$ & $75 \mathrm{y}$ & $72 \mathrm{y}$ & $71 \mathrm{y}$ \\
\hline $\begin{array}{l}\text { Sex } \\
\text { distribution }\end{array}$ & & & \\
\hline Male & $18(70 \%)$ & $20(63 \%)$ & $16(55 \%)$ \\
\hline Female & $8(30 \%)$ & $12(37 \%)$ & $13(45 \%)$ \\
\hline
\end{tabular}

Abbreviations: MRPI, magnetic resonance Parkinsonism index; PD, Parkinson's disease; PSP, progressive supranuclear palsy.

Table 2 Mean with standard deviation of various MR planimetric measurements in our study population

\begin{tabular}{|l|l|l|l|}
\hline Variables & PSP $(\boldsymbol{n}=\mathbf{2 6})$ & PD $(\boldsymbol{n}=\mathbf{3 2})$ & Control $(\boldsymbol{n}=\mathbf{2 9})$ \\
\hline $\begin{array}{l}\text { Pons } \\
\text { area } \\
\left(\mathrm{cm}^{2}\right)\end{array}$ & $\begin{array}{l}4.8950 \pm \\
0.55999\end{array}$ & $\begin{array}{l}4.7384 \pm \\
0.82279\end{array}$ & $\begin{array}{l}4.4648 \pm \\
0.64571\end{array}$ \\
\hline $\begin{array}{l}\text { Midbrain } \\
\text { area } \\
\left(\mathrm{cm}^{2}\right)\end{array}$ & $0.9412 \pm$ & $\begin{array}{l}1.3116 \pm \\
0.28216\end{array}$ & $\begin{array}{l}1.5817 \pm \\
0.31195\end{array}$ \\
\hline $\begin{array}{l}\text { Width } \\
\text { of MCP } \\
(\mathrm{cm})\end{array}$ & $1.3354 \pm$ & $0.9272 \pm$ & $1.1700 \pm$ \\
& & 0.23317 & 0.19860 \\
Width & $0.3735 \pm$ & $0.3119 \pm$ & $0.4524 \pm$ \\
of SCP & 0.10480 & 0.05954 & 0.08279 \\
$(\mathrm{~cm})$ & & & \\
\hline P/M & $5.3073 \pm$ & $3.7294 \pm$ & $2.8793 \pm$ \\
& 0.94920 & 0.83307 & 0.49974 \\
\hline MCP/ & $3.7015 \pm$ & $2.9534 \pm$ & $2.6245 \pm$ \\
SCP & 0.87366 & 0.49913 & 0.43940 \\
\hline
\end{tabular}

Abbreviations: MCP, middle cerebellar peduncle; SCP, superior cerebellar peduncle SCP.

Patients with PSP exhibited less value of midbrain area ( - Table 2 and - Fig. 3a-c) when compared with the other two groups with the mean value of 0.9 and also found to be very significant with $p$-value $<0.001$, which points to the main pathology of midbrain atrophy in PSP. ${ }^{1}$

Meanwhile, there is no statistical significance noted in the Pons area among the three groups ( $p$-value 0.07) (-Table 2).

Patients with other PD show highly significant difference in the mean value of width of middle cerebellar peduncle $(0.9 \pm 0.3 ; p$ value $<0.001)$ as middle cerebellar peduncle atrophy can occur in multisystem atrophy disease (-Table 2 and -Fig. $4 a-c){ }^{4}$

Several studies prove the atrophy of SCP in PSP patients, but in our study no significant results related to it were noted (-Table 2). ${ }^{5}$

Patients with PSP were also found to have cardinal findings ( $p$-value of $<0.001$ ) with the parameter $\mathrm{M} / \mathrm{P}$ ratio (lower in PSP patient with mean value of $0.18 \pm 0.036$ ) and $\mathrm{P} / \mathrm{M}$ ratio (higher in PSP patients with mean value of $5.3 \pm 0.94$ ), which
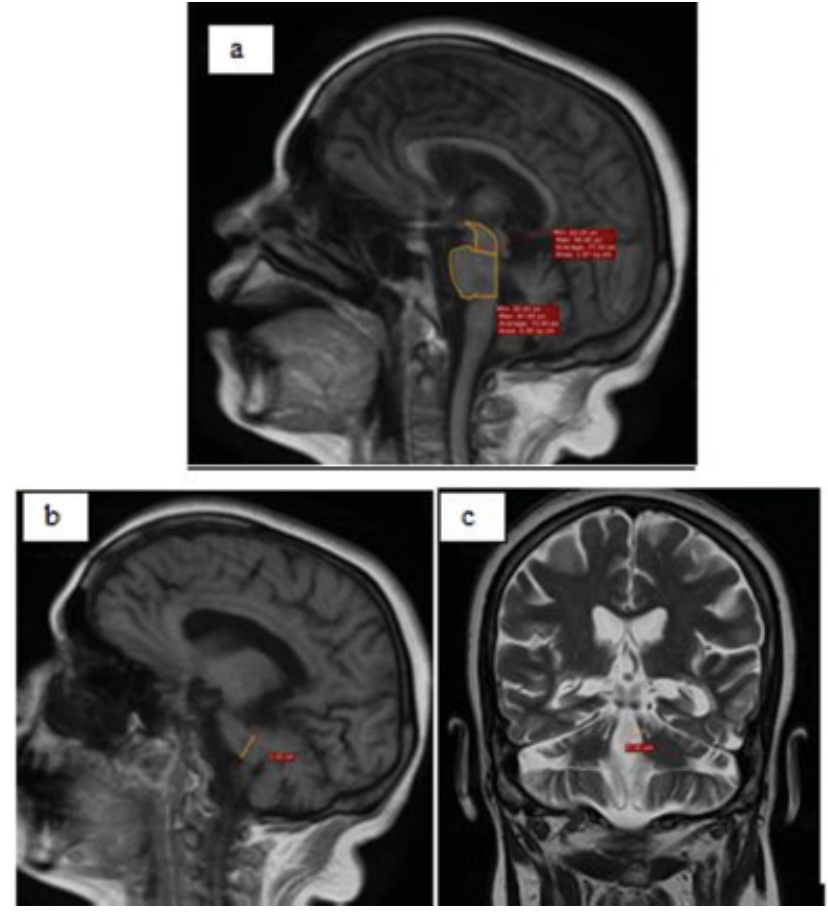

Fig. 3 T1W sagittal image of progressive supranuclear palsy patient (a), calculating the area of midbrain and pons which was 1.07 and 4.35 , respectively. $\mathrm{P} / \mathrm{M}$ ratio $=4.1 ; \mathrm{M} / \mathrm{P}$ ratio $=0.24$. T1W sagittal $(\mathrm{b})$ and $\mathrm{T} 2 \mathrm{~W}$ coronal (c) of progressive supranuclear palsy patient showing the width of MCP (1.22) and SCP (0.16).
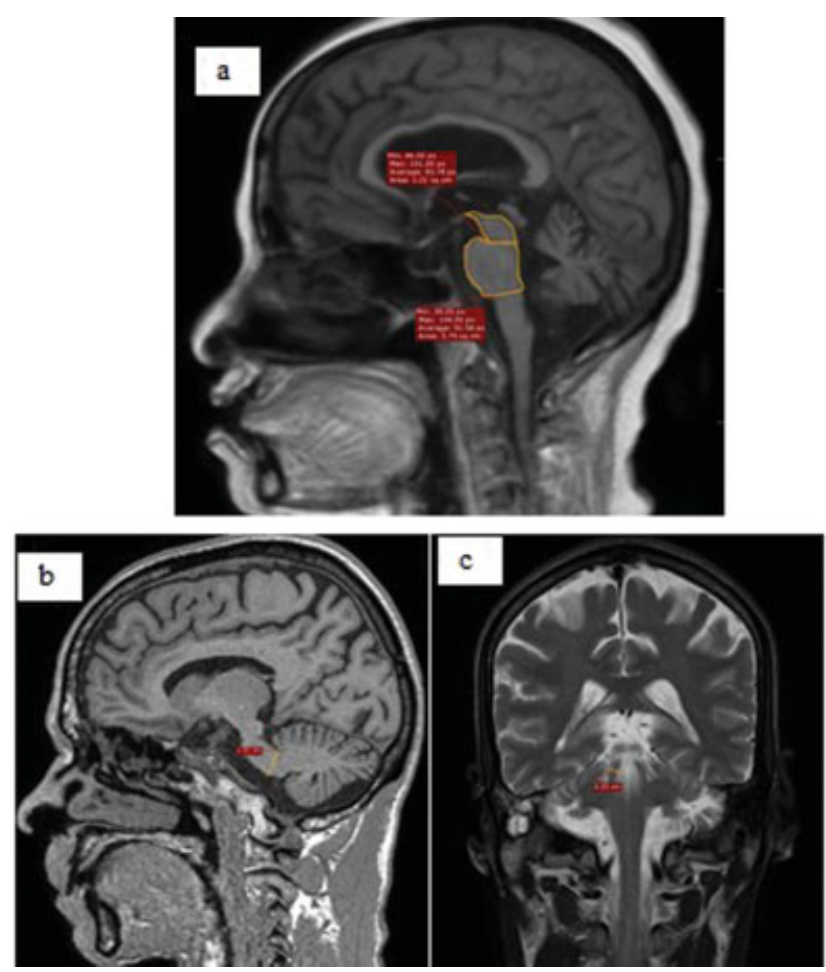

Fig. 4 T1W sagittal image of Parkinson's disease patient, calculating the area of midbrain and Pons (a) which was 1.21 and 3.79,

respectively. $\mathrm{P} / \mathrm{M}$ ratio $=3.15 ; \mathrm{M} / \mathrm{P}$ ratio $=0.32$. T1W sagittal $(\mathbf{b})$ and T2W coronal (c) of Parkinson's disease patient showing the width of MCP (1.3) and SCP (0.33). 
Table 3 Mean with standard deviation of M/P ratio and MRPI index in PSP, PD, and control groups

\begin{tabular}{|l|l|l|l|}
\hline Variable & PSP $(\boldsymbol{n}=\mathbf{2 6})$ & PD $(\boldsymbol{n}=\mathbf{3 2})$ & Control $(\boldsymbol{n}=\mathbf{2 9})$ \\
\hline M/P & $0.1838 \pm$ & $0.2766 \pm$ & $0.3531 \pm$ \\
& 0.03678 & 0.05469 & 0.05419 \\
\hline MRPI & $19.5077 \pm$ & $10.4503 \pm$ & $7.5000 \pm$ \\
& 5.7862 & 1.22262 & 1.50751 \\
\hline
\end{tabular}

Abbreviations: MRPI, magnetic resonance Parkinsonism index; PD, Parkinson's disease; PSP, progressive supranuclear palsy.

Table 4 Cut-off value of MRPI index in PSP, PD vs. control groups with their corresponding sensitivity and specificity

\begin{tabular}{|l|l|l|l|}
\hline MRPI & Cut-off value & Sensitivity & Specificity \\
\hline PSP vs. PD & 13.4 & $100 \%$ & $100 \%$ \\
\hline PSP vs. control & 12.35 & $100 \%$ & $100 \%$ \\
\hline PD vs. control & 9.025 & $91 \%$ & $83 \%$ \\
\hline
\end{tabular}

Abbreviations: MRPI, magnetic resonance Parkinsonism index; PD, Parkinson's disease; PSP, progressive supranuclear palsy.

also indicates that there is atrophy of midbrain in PSP patients.

The mean MRPI value was higher in almost all patients of PSP patients compared with PD and controls as described in (-Table 3). MRPI value for PD and control patient was found to be less than 12 (PD: 10.4 and control: 7.5) with no overlapping value with PSP patients, which was found to be 19.5 .

The cut-off value of MRPI was fixed as 13.4 (-Table 4) using ROC curve analysis (-Fig. 2a-c) with $100 \%$ sensitivity and $100 \%$ specificity in case of PSP versus PD group and 12.3 in PSP versus control group.

Although the M/P ratio was found to be statistically significant in PSP patients ( $p$-value $<0.0001$ ) and the mean value (approximately 0.2 ) is less compared with the other two groups ( - Table 3 ), the cut-off value could not be made out as the values are very low, and thus MRPI index is deduced as a good predictor for the quantitative assessment of PSP.

\section{Discussion}

Parkinsonism plus syndromes are a group of neurodegenerative disorder under which are categorized the PSP, multisystem atrophy, and corticobasal degeneration. As a group, they present with symptoms of Parkinsonism (rigidity and bradykinesia), but typically have some slightly different clinical picture. $^{5}$

PSP is a form of atypical Parkinsonism which is characterized by slow ocular saccades, eyelid apraxia, and restricted eye movements with particular impairment of downward gaze. In later stages, speech and swallowing difficulty and dementia become evident. ${ }^{1}$

Multisystem atrophy manifest as a combination of parkinsonian, cerebellar, and autonomic features and can be divided into a predominant parkinsonian form.

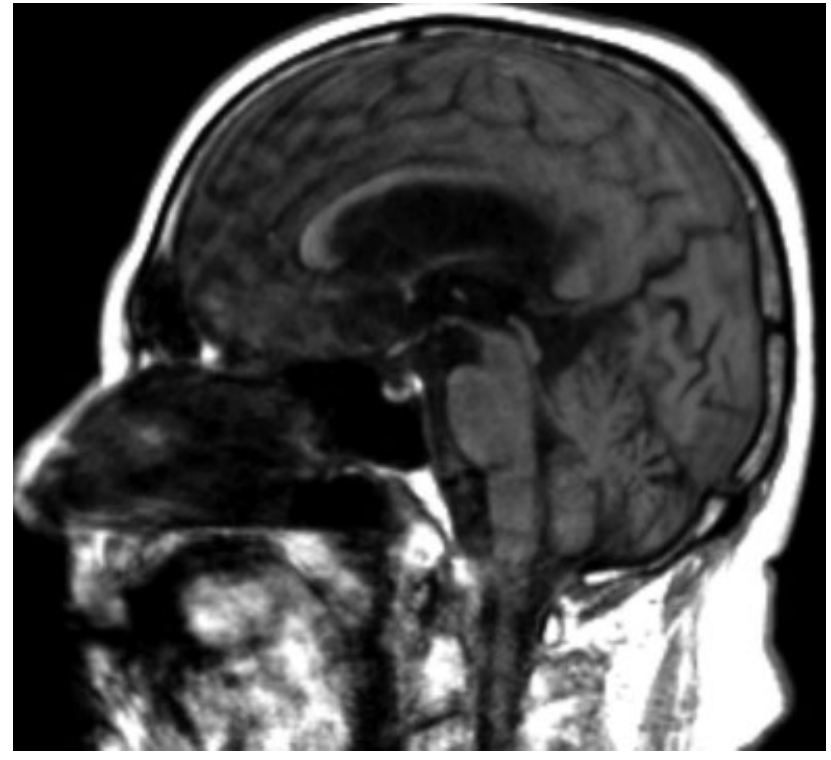

Fig. 5 MRI T1W sagittal image shows Hummingbird sign in a case of progressive supranuclear palsy (PSP).

Corticobasal degeneration is rare and usually manifest by asymmetric dystonic contractions and clumsiness of one hand coupled with cortical sensory disturbances manifest as apraxia, agnosia, focal myoclonus, or alien limb phenomenon. ${ }^{6}$

Atypical Parkinsonism spectrums are associated with degeneration of dopamine neurons. Neuroimaging of the dopamine system is usually not helpful in the diagnostic purpose. ${ }^{7}$

MRI forms the mainstay of imaging modality for these conditions. Even though some qualitative markers like Humming bird sign (-Fig. 5) (seen in mid sagittal images due to atrophy of midbrain with preservation of Pons), morning glory sign (due to widening of interpeduncular cistern with lateral concavity of midbrain) and Mickey Mouse sign are specific for PSP to differentiate from other PD, these are mostly observed in the late stages of the disease. ${ }^{5}$ So, for early distinction of PSP, some quantitative markers like MRPI and midbrain/pons ratio $(\mathrm{M} / \mathrm{P})$ are useful. ${ }^{8,9}$

In our study results, the mean value of midbrain area was statistically significant in PSP patients compared with the other two groups (PD and control), which was similar in comparison to the study done by Zanigni et $\mathrm{al}^{10}{ }^{10}$ where the area of midbrain was found to be the most accurate significant diagnostic marker with $96 \%$ sensitivity and $98 \%$ specificity than MRPI, where they obtained a cut-off value as $\geq 10.67$ with $87 \%$ sensitivity and $93 \%$ specificity.

Another significant remark of PSP is the atrophy of midbrain, which is pointed by our quantitative results of higher $\mathrm{P} / \mathrm{M}$ and lower $\mathrm{M} / \mathrm{P}$ ratio in PSP patients.

On plotting the ROC curve, the cut-off value of MRPI was obtained as 13.4 with $100 \%$ sensitivity and specificity from our study ( - Fig. 2a-c and - Table 4 ). Although M/P ratio was found to be statistically significant, cut-off values were very low.

In a study conducted by Constantinides et al, ${ }^{4}$ the cut-off value of MRPI was achieved as 12.6 with $91 \%$ sensitivity and 95\% specificity and the cut-off value for $\mathrm{M} / \mathrm{P}$ ratio was obtained as $<0.22$ with $88 \%$ sensitivity and $84 \%$ specificity. 
Among the three parameters which they included in their study (MRPI, M/P ratio, and M/CC), MRPI was established as the best parameter for the diagnosis of PSP.

Nigro et al $^{11}$ study compared the manual and automatic method of calculation of MRPI and concluded with a cut-off value of MRPI for PSP patients as >13.42 with almost 93\% sensitivity and $100 \%$ specificity.

Quattrone et $\mathrm{al}^{2}$ concluded that MRPI index along with combined assessment of routine MR imaging can help differentiate patients with PSP from those with PD, with a cutoff value of $\geq 13.5$ ( $100 \%$ sensitivity and specificity).

\section{Limitations}

- The study was conducted with less number of samples.

- Three-dimensional T1W sequences were not obtained as routine investigation.

- Interobserver correlation was not obtained, as they were not blinded.

\section{Conclusion}

From this study, MRPI index has ascertained to be additional quantitative marker in diagnosing PSP patients with a cut-off value of MRPI as 13.4 in comparison to $\mathrm{M} / \mathrm{P}$ ratio. Some parameters like midbrain area, Pons/midbrain ratio, and $\mathrm{M} / \mathrm{P}$ are also useful for the diagnosis of PSP. In conclusion, combined qualitative as well as quantitative measurement of MRPI will further improve diagnosing PSP.

\section{Financial Support and Sponsorship}

None.

\section{Conflict of Interest}

None declared.

\section{Acknowledgment}

We acknowledge the Department of Neuro Medicine and Community Medicine for extending their support throughout the study

\section{References}

1 Quattrone A, Morelli M, Williams DR, et al. MR parkinsonism index predicts vertical supranuclear gaze palsy in patients with PSP-parkinsonism. Neurology 2016;87(12):1266-1273

2 Quattrone A, Nicoletti G, Messina D, et al. MR imaging index for differentiation of progressive supranuclear palsy from Parkinson disease and the Parkinson variant of multiple system atrophy. Radiology 2008;246(01):214-221

3 Lake H, Tomazzoni T, Muzio BD, Gaillard F. Top 10 Helpful Measurements and Scoring System for Neurodegenerative Brain MRI Interpretation. EPOS; 2015:1-14

4 Constantinides VC, Paraskevas GP, Velonakis G, Toulas P, Stamboulis E, Kapaki E. MRI planimetry and Magnetic Resonance Parkinsonism Index in the differential diagnosis of patients with parkinsonism. AJNR Am J Neuroradiol 2018;39(06): 1047-1051

5 Sankhla CS, Patil KB, Sawant N, Gupta S. Diagnostic accuracy of Magnetic Resonance Parkinsonism Index in differentiating progressive supranuclear palsy from Parkinson's disease and controls in Indian patients. Neurol India 2016;64(02):239-245

6 Whitwell JL, Höglinger GU, Antonini A, et al; Movement Disorder Society-endorsed PSP Study Group. Radiological biomarkers for diagnosis in PSP: where are we and where do we need to be? Mov Disord 2017;32(07):955-971

7 Aydn E, Eraslan C, Acarer A, et al. The Morphometric Parameters in MRI for Differentiation Progressive Supranuclear Palsy from Parkinson's disease, Multiple System Atrophy and Controls. EPOS; 2017:1-11

8 Zhang K, Liang Z, Wang C, Zhang X, Yu B, Liu X. Diagnostic validity of magnetic resonance parkinsonism index in differentiating patients with progressive supranuclear palsy from patients with Parkinson's disease. Parkinsonism Relat Disord 2019; 66:176-181

9 Nigro S, Morelli M, Arabia G, et al. Magnetic Resonance Parkinsonism Index and midbrain to pons ratio: which index better distinguishes Progressive Supranuclear Palsy patients with a low degree of diagnostic certainty from patients with Parkinson Disease? Parkinsonism Relat Disord 2017;41:31-36

10 Zanigni S, Calandra-Buonaura G, Manners DN, et al. Accuracy of MR markers for differentiating Progressive Supranuclear Palsy from Parkinson's disease. Neuroimage Clin 2016;11:736-742

11 Nigro S, Arabia G, Antonini A, et al. Magnetic Resonance Parkinsonism Index: diagnostic accuracy of a fully automated algorithm in comparison with the manual measurement in a large Italian multicentre study in patients with progressive supranuclear palsy. Eur Radiol 2017;27(06):2665-2675 